\title{
PRESERVING NATIONAL SLANG WITH MOBILE TECHNOLOGY
}

\author{
Fatima Saleh Al Dhaen ${ }^{1 *}$
}

*1Ahlia University Information Technology Dep. P.O. Box 10878, 1st Floor, Gosi Complex, Exhibitions Road, Manama Kingdom of Bahrain faldhaen@ahlia.edu.bh; fatima998877@yahoo.com

*Corresponding Author: -

Email ID: - faldhaen@ahlia.edu.bh; fatima998877@yahoo.com

\begin{abstract}
: -
Arabic language and national slangs are really in danger since they ae starting to fade lately due to the globalization era that we are facing nowadays; English has become the most dominating language in the Middle East since it becomes the lingua franca of education, media and commerce particularly in the gulf region. Unfortunately, the misconception of not using national slangs in daily live conversations is very noticeable due to the new western educational systems and media outlets. Preserving national slangs is very crucial and important to protect our language from vanishing by using mobile technology to teach the indigenous people their national slangs and even those who are expatriates who wish to learn a certain national slang through mobile learning.

The main purpose of this paper is to demonstrate a new free educational application called Bahraini slang dictionary based on Bahraini slang only, BSD comes with two languages (Arabic and English) in addition to audio pronunciation.
\end{abstract}

Keywords: - HCI, Diglossia, MALL, M-Learning, OS, lingua franca 


\section{INTRODUCTION}

In the twenty first century, teaching and learning environments have been changed so drastically due to extraordinary prospects progression of information and communication tools have created for education especially after the speedy development and evolution of smart devices which opened up new opportunity for new technological learning tools and experiences.

Nowadays they are plenty of free educational applications that give the user the ability to learn, listen and practice through words and phrases which ease and facilitate the comprehension of learning. Each country has its own cities and outskirts traditions, rituals, slangs and epigrams. Learning other countries urban slang would be a great positive and beneficial idea to be able to socialize with local suburban.

Kingdom of Bahrain is an island country consisting of a small archipelago centered on Bahrain Island; it has a very rich history in more than 32 small islands, it consists of many cities and villages; each suburb has its own slang and every slang has its own words, phrases, vocabularies and proverbs.

\section{Problem statement}

Kingdom of Bahrain is a very modern and civilized country that has a very high educational system whether in governmental or private sectors. The education system of Bahrain always ensures using the best techniques and performances to facilitate the role of understanding and comprehension.

Regrettably teenagers tend to be shy and try not to speak their national slangs and not even try to learn it from their parents and their grannies and prefer to speak English since it becomes compulsory language and one of the main international languages that become a trend to brag of using it in their daily lives.

The government of Bahrain always emphasizes on the importance of Arabic language because it's the main language of the holy Quran and it's considered as the main cornerstone of Arab nationalism. The issue has been discussed through many international conferences, debates and always on the top agendas of the general meeting of Shure or parliament councils therefore despite all the efforts done by ministry of education, issue is still ongoing and hasn't been tackled yet. Arabic somehow is slightly evaporated and fading out especially for those who study in private schools or abroad (boarding schools) or socialize with western communities.

Not only Arabic language that has been disappearing but also national slang of any country. National Slang that we have inherited from our ancestors and dynasties should be well- preserved and learned through generations. No one can deny the importance of acquiring new languages to be fluent speaker with no single obstacle however any countries slang would be a heritage and great legacy that we must preserve and teach our children those treasured words and proverbs.

The reason behind eliminating Arabic or national slang is because Arabic diglossia that identified as bilingual situation of changing the concentration in which the continuum finds itself in a forceful conflictual diversity with foreign languages such as English, French \& Farsi in some Arab countries.

Most of Arabic language teachers in Bahrain whether in government or private school come from different regions with different spoken dialects and that really affects the national education in the country by spreading their dialects, culture and accent to Bahraini students.

"Even though the problem of Arabic diglossia seems to have attracted the attention of Western sociolinguists, many Arab linguists have given it attention in the last three decades."

\section{Smartphone addiction}

Since smartphone technologies with free countless educational applications show the effectiveness methods of learning and gaining information with very accessible and easy ways.

Over the past years, mobile learning has developed enormously in educational organizations and institutions as well as societies. It started with simple text based short lessons sent over to mobile devices for revision or a quick recap; nowadays M-learning is increasing in scope and ability that offer new chances for gaining knowledge that extend beyond the basic traditional e -Learning or instructor-led classroom.

The addiction of the using smart-devices and the internet been escalating day after day especially for those teenagers who really rely on their mobiles $24 / 7$ whether for education purposes or sending emails, entrainment, etc.

Mobile applications can be accessed and used without any telecommunication services once it been installed in the device.

\section{Assistant tools}

Mobile Assisted Language Learning (MALL) has been identified as a new tool of language learning that performs with the assistance of portable devices. "MALL differs from computer-assisted language learning in its use of personal, portable devices that enable new ways of learning, emphasizing continuity or spontaneity of access and interaction across different contexts of use".[1]

\section{BSD (Bahraini slang dictionary)}

BSD (Bahraini slang dictionary), it's a free application to be found in Play store and installed freely with $0 \%$ charges to allow those Bahrainis who haven't learned Bahraini slangs well or for non-Bahrainis who would be interested to learn the slang through English translation.

BSD helps those who would like to learn English language through Bahraini slang by typing the word in the typing bar, and the word comes instantly with the description of the specific word or proverb in both languages (Arabic -English) in 
addition to audio pronunciation system, if a person wants to pronounce or utter the word correctly he/she can click on speaker's button to listen to the right pronunciation in Bahraini slang or English language.

\subsection{The Model used}

"In this project the iterative classical waterfall model been selected because of the "facility of giving feedback to the previous stage, with the help of this facility it is easy to rectify error before going to next stage. Since error propagates to backward that's why it is called iterative waterfall model."

The main advantages of using iterative classical waterfall model

1- It's very simple model especially for those have well understood goals.

2- Errors and bugs can be rectified and tackled easily.

3- Stages are pretty defined

4- The implantation process is very flexible and easy stage to be processed."[2]

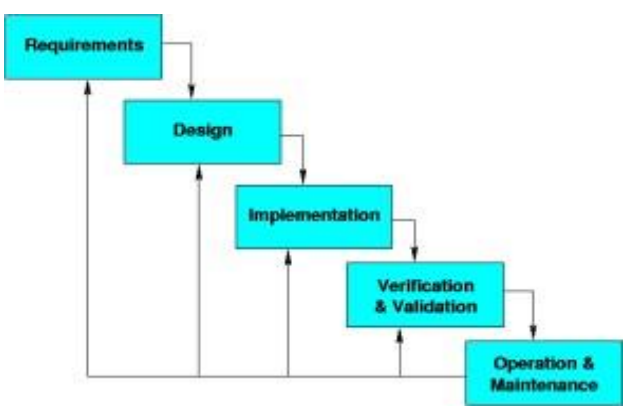

Figure 1 [iterative waterfall model]

https://secweb.cs.odu.edu/ zeil/cs350/f14/Public /processModels/index.html

5.1.1 Requirement "Since it's a mobile application based on Android system because it's a commonly used operating system and it has an inclusive platform focused in smart devices, it contains a set of software on a flexible level combined by an OS, middleware, and applications.

There main reasons of selection Android operating system for developing mobile interactive applications as you find below: -

- Completely free platform using Linux based system which permits the usage of its APIs (Application Programming Interface) without speculation

- It provides a good API system that contains rich accessibility assets.

- Android application ships with a set of core applications client, SMS program, calendar, maps, browser, contacts and others, all these applications are developed in java

- It is based on the Java programming language (Eclipse), which is one of the most prominent and easy language used in programming developing era." [3]

Adobe illustrator been used to sketch the BSD mobile application pages that will briefly shown then the rest of the buttons and images were designed by Adobe Photoshop.

Bahraini flag was chosen as the main interface to be a good representation of patriotism which will be displayed as the main gateway of the application. Simplicity is very important when it comes to HCI rules, so red, white and black color been

\subsubsection{System Design and user interface}

\subsubsection{Human computer interaction}

Human computer interaction is a very crucial and important stage especially for designing because visibility, clearance, mixing colors, flawless of any bugs or errors, ambiguousness, type of users whether the user is normal or with disability have to be taken into consideration to make the designated project very user friendly to reach the target audience.

"HCI (human-computer interaction) is the study of how people interact with computers and to what extent computers are or are not developed for successful interaction with human beings. HCI term helps people with different mental models, culture, knowledge, and other differences to contribute with the computer and enhance it to be more userfriendly". [4]

HCI needs to take into concern of the social, political, ethical, and societal implications of computer systems. [5]

\subsubsection{BSD staring page}

The background of the main interface been selected as Bahraini flag as sign of patriotism which shows the logo of the BSD and the name of the application as Bahrain Slang Dictionary 


\subsubsection{Design}

Adobe illustratorbeen used to sketch the BSD mobile application pages that will briefly shown then the rest of the buttons and images were designed by Adobe Photoshop. Bahraini flag was chosen as the main interface to be a good representation of patriotism which will be displayed as the main gateway of the application.Simplicity is very important when it comes to HCI rules, so red, white and black color been chosen as the main Background color of searching and typing the words in the searching bar.Smartphone gives the opportunity to flip the keyboards to any languages user might select.

\subsubsection{HCI Users Diversity}

The first key of developing a human-computer interface is to recognize the diversification of human mental knowledge, or background. There are three types of people that may access your HCI applications, system, website or game. But the most important people that you need to take into concern about are the novices. Novices are those who are newly using computers or mobile applications and will need to seek the help in order to interact with it. The second diversity are the intermittent user, they don't lack the knowledge but can deal with parts of the interaction. The last but not least are the expert which has wide background and uses the computers rapidly and all they need is to get things done as they want. One needs to take those diversifications into concern and design an interaction that is compatible to all. [6]

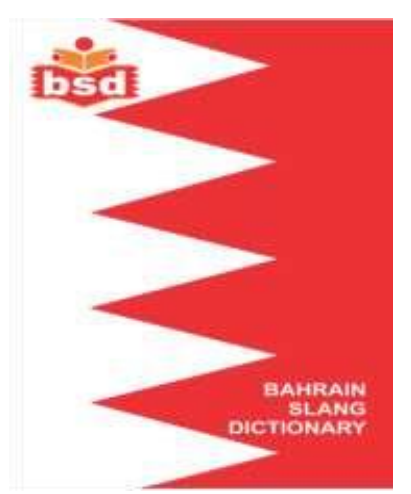

Figure 2 Main Interface

\section{Main Menu}

The main menu of the BSD interface has 4 buttons displayed in both languages English and Arabic language Those 4 icons are clickable once the user clicks on them, the designated page will be shown instantly

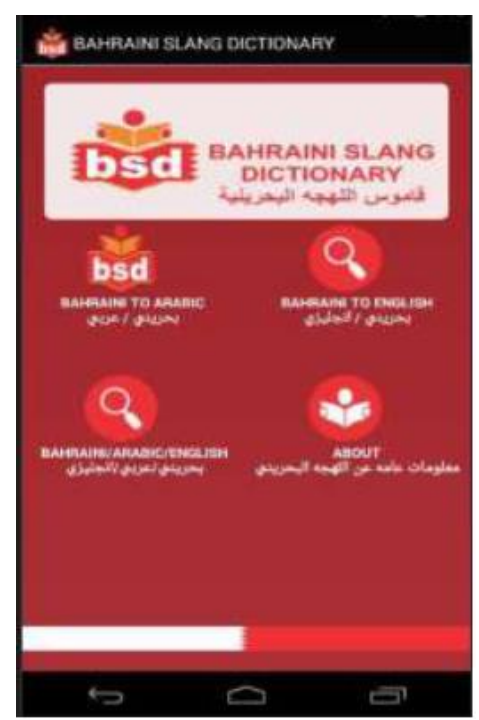

Figure 3: BSD Structure 


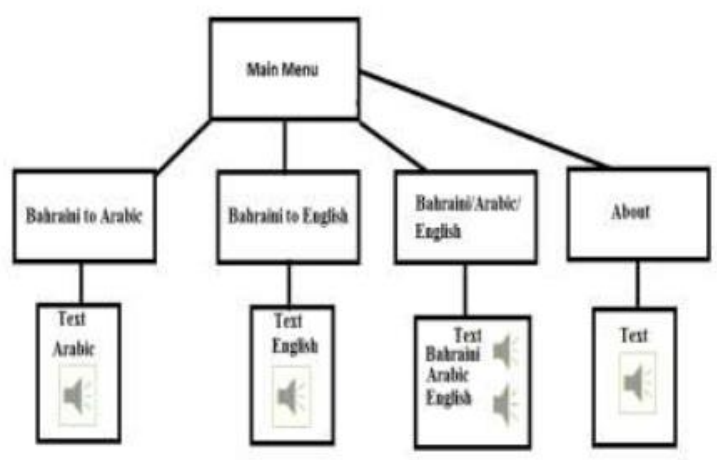

Figure 4: Menu Icons

\section{Bahraini to Arabic:}

This icon takes the user to a page with a typing bar so anyone can type the word he/she desires, and the Arabic description will be displayed with voice over.

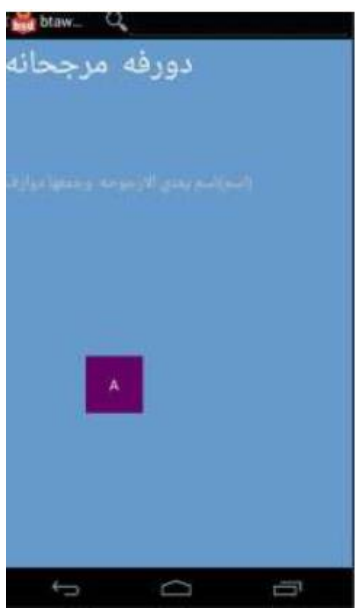

Figure5: Arabic/Bahraini slang translation

\section{Bahraini to English:}

This icon takes the user to a page with a typing bar so user can type the word he desires and then English description will be displayed with voice over. Of both the slang and English word for those who would love to learn English through this app and it shows the type of the word for example if it's a verb, noun, adjective etc.

- The letter A indicates Arabic -user can listen to the Arabic-Bahraini slang pronunciation

- The letter E indicates English -user can listen to the English pronunciation

\section{- Bahraini/Arabic/ English}

This icon takes the user to page where all the 2 main languages Arabic and English in addition the Bahraini slang will be displayed together with voice pronunciations

- About: This icon takes the user to the information page to enlighten him/her of Bahraini slang types and how to put words in the right sentences or context with voice over.

- User has the option to read or to listen to the full text provided 


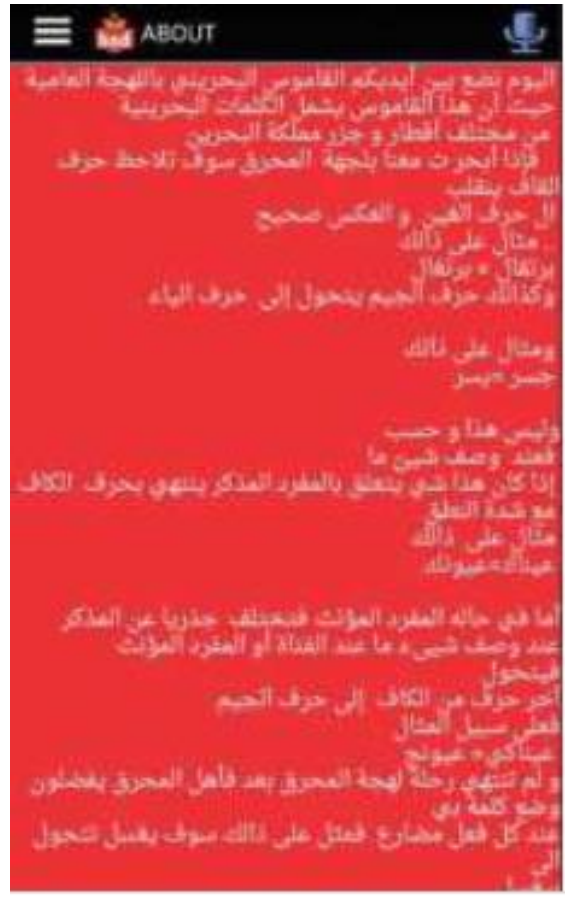

Figure6 About (Information)

\subsubsection{Implantation}

\section{- Building and coding the system}

Coding phase translates proposed design of the system into codes using certain programming language. The goal of the stag is to implement the design in the finest possible method. The coding stage affects both testing and maintenance profoundly.

Since Google's Android operating system uses Java as the basis for all Android apps so Java was used to build BSD application due to simplicity and clarity of using it.

\subsubsection{Verification and validation}

\section{- Testing through Quantitative Research}

"Quantitative Research was driven by investigators with the need to quantify data. Since then quantitative research has dominated the western cultural as the research method to create meaning and new knowledge. What constitutes a quantitative research method involves a numeric or statistical approach to research design.

"Several research methods exist to conduct quantitative research. In descriptive research method, correlational, developmental design, observational studies, and survey research are used. These research methods may also be used in various degrees with experimental and causal comparative research".[7]

Quantitative research is easier to get statistics as well as retrieving the data in future for business requirements or further references. In my opinion this method of research is saves much more time than the qualitative research as well as quantitative methodology allows the individual to be more biased and feel free to express without prolonging their answers.

Quantitative research methods are mostly controversial between the two schools, where the methods are linked directly to the classical scientific paradigm. Quantitative methods are basically gathering complete data or information, for instance gathering numerical statistics will result in providing clear and fair outcomes. Nonetheless, there are many other methods that could affect the quantitative researches and make them as clear as it should be. Furthermore, this method basically comes after the research project is done and well understood. The main reason for quantitative research is to have the ability to spread information and categorize them according to the project plan and make it easier to handle numerical information. The authorized personnel are responsible for clarifying the information and keep it measured as much as possible. Generally, the purpose of quantitative researches is for the subject of analyzing statistics and making them accessible and understandable.

Thirty students were chosen to use the BSD application and at the same time a questionnaire (was conducted and allocated to them. I chose this type of analysis since it can be measured. This is found by the calculating the answers of categorized question. 


\begin{tabular}{|c|c|c|c|}
\hline \multicolumn{4}{|l|}{$\begin{array}{l}\text { Please rate the Application according to the following classifications: } \\
\text { 3. Good 2. Fair 1. Needs of improvement }\end{array}$} \\
\hline & 3 & 2 & 1 \\
\hline \multicolumn{4}{|l|}{ BSD application Content } \\
\hline \multicolumn{4}{|l|}{ 1. Is the content clear? } \\
\hline \multicolumn{4}{|l|}{ 2. Does the BSD application contain all the required information? } \\
\hline \multicolumn{4}{|l|}{ 3. The arrangement of information content on the BSD application is clear? } \\
\hline \multicolumn{4}{|l|}{ 4. Are there any mistakes (spelling, grammar, etc...)? } \\
\hline \multicolumn{4}{|l|}{ BSD application Usability } \\
\hline \multicolumn{4}{|l|}{ 1. Do you find the BSD application easy to navigate? } \\
\hline \multicolumn{4}{|l|}{ 2. Does the BSD application load fast? } \\
\hline \multicolumn{4}{|l|}{ 3. Is it easy to find required information in the BSD application? } \\
\hline \multicolumn{4}{|l|}{ 4. The graphics/designs on the BSD application are pleasant? } \\
\hline \multicolumn{4}{|l|}{ 5. As a first time user, do you find it easy? } \\
\hline \multirow{2}{*}{\multicolumn{4}{|c|}{$\begin{array}{l}\text { 6. Clicking on a button takes you to the required information? } \\
\text { BSD application Desion }\end{array}$}} \\
\hline & & & \\
\hline \multicolumn{4}{|l|}{ 1. Do you find an integration of design in the BSD application? } \\
\hline \multicolumn{4}{|l|}{ 2. Is the BSD application visually appealing? } \\
\hline \multicolumn{4}{|l|}{ 3. Is the BSD application font readable/clear? } \\
\hline \multicolumn{4}{|l|}{ 4. Are the WebPages designs confusing/over exaggerated? } \\
\hline \multicolumn{4}{|l|}{ 5. Are the colors used readable? } \\
\hline \multicolumn{4}{|l|}{ 6. Is the font color compatible with the background color? } \\
\hline \multicolumn{4}{|l|}{ Audio Buttons Functionality } \\
\hline 1.Are the audio buttons both Arabic and English are working fine & & & \\
\hline \multicolumn{4}{|l|}{ Overall } \\
\hline \multicolumn{4}{|l|}{ 1. How useful is the BSD application? } \\
\hline \multicolumn{4}{|l|}{ 2. How accurate is the BSD application? } \\
\hline 3. How easy it is to deal with? & & & \\
\hline Suggestions for Improvements: & & & \\
\hline
\end{tabular}

\section{Survey 1: Survey questions}

\begin{tabular}{|l|l|}
\hline Gender & Responded \\
\hline Male: & 15 \\
\hline Female: & 12 \\
\hline Total: & 27 \\
\hline
\end{tabular}

Table 1: Survey Reponses

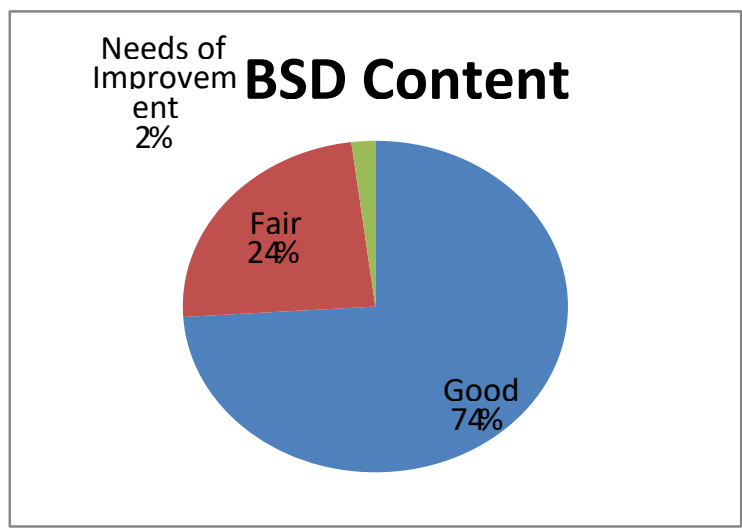

Figure 7 BSD Application Content Analysis - Pie Chart

The application content questions cover the richness of information, the clearance of information, phrases and the mistakes found on the content. The above figure shows the total results of website content; one can assume that it's good in this term since it ranked $74 \%$. The website content got fair $24 \%$ and needs of improvement $2 \%$ as seen in figure 4.1 . 


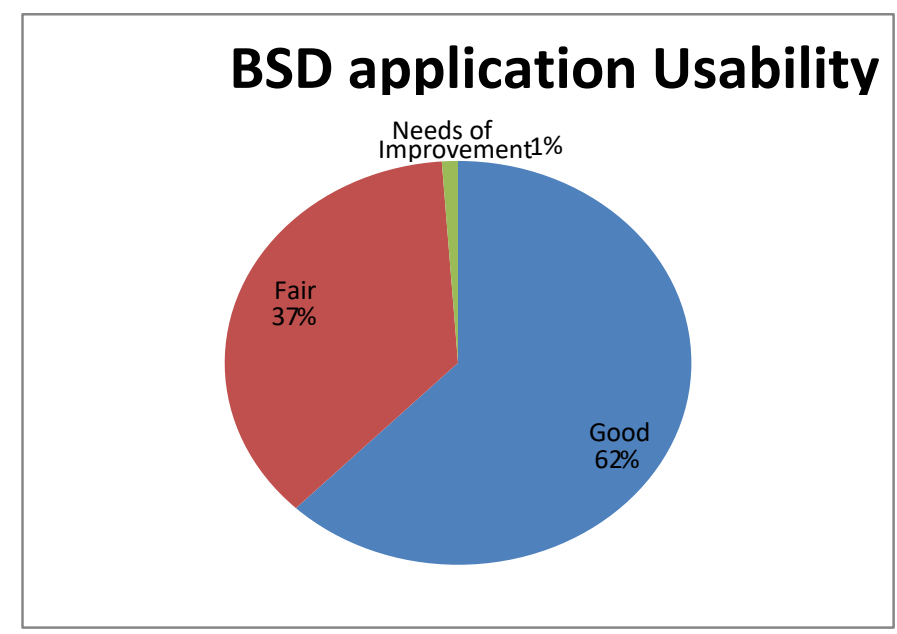

Figure 8 BSD Application Usability Analysis - Pie Chart

Figure 8 shows the results of website was good since it got $62 \%$, fair $37 \%$ and needs of usability in terms of how easy to use, navigate, improvement $1 \%$. pleasing, and obtaining. The website usability was good since it got $62 \%$, fair $37 \%$ and needs of improvement $1 \%$.

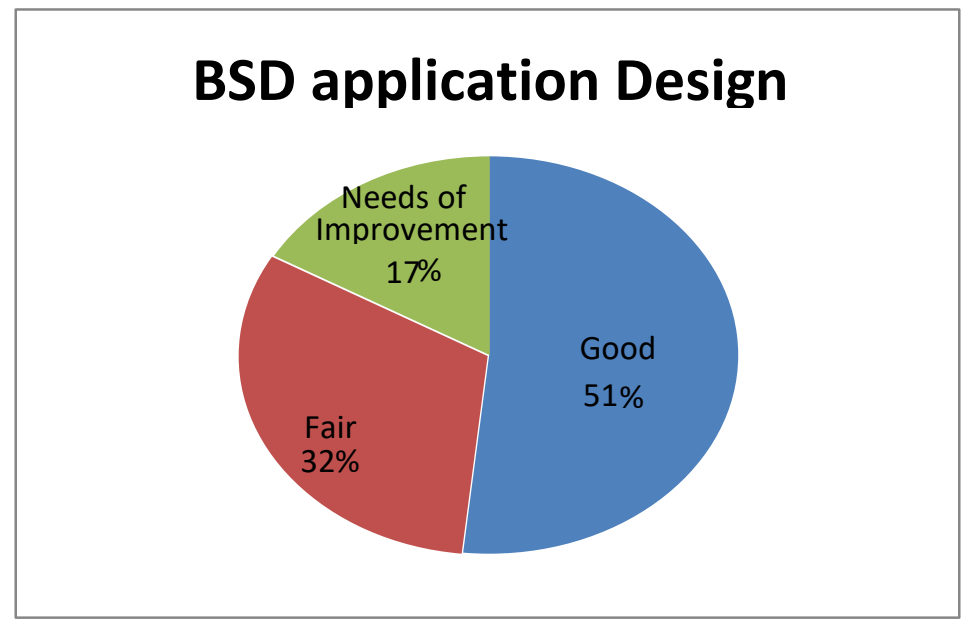

Figure 9 BSD Application Design Analysis - Pie Chart

The BSD application design shows the results of good, fair $32 \%$ and needs of improvement $17 \%$ as the design in terms of colors, font, and the visual clearly seen in figure 9. appealing of the application It's ranked $51 \%$ good, fair $32 \%$ and needs of improvement $17 \%$ as clearly seen in figure 9 .

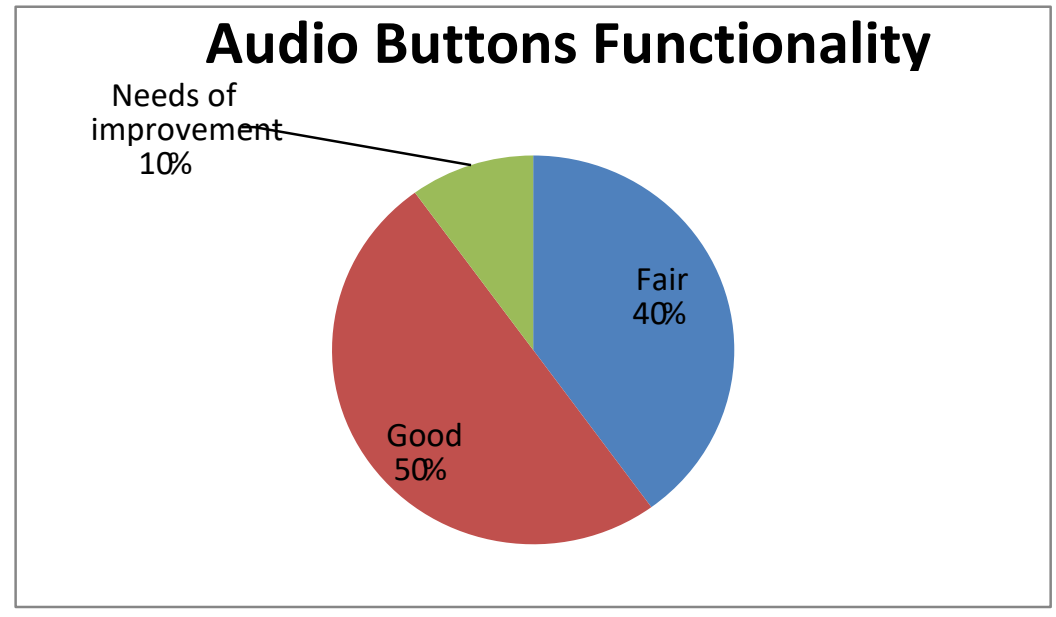

Figure 10 - BSD Audio Buttons Functionality-part chart

The BSD application design shows the results of the audio buttons functionality in terms of hearing process of the application, It's ranked 50\% good, fair $40 \%$ and needs of improvement $10 \%$ as clearly seen in figure 11 


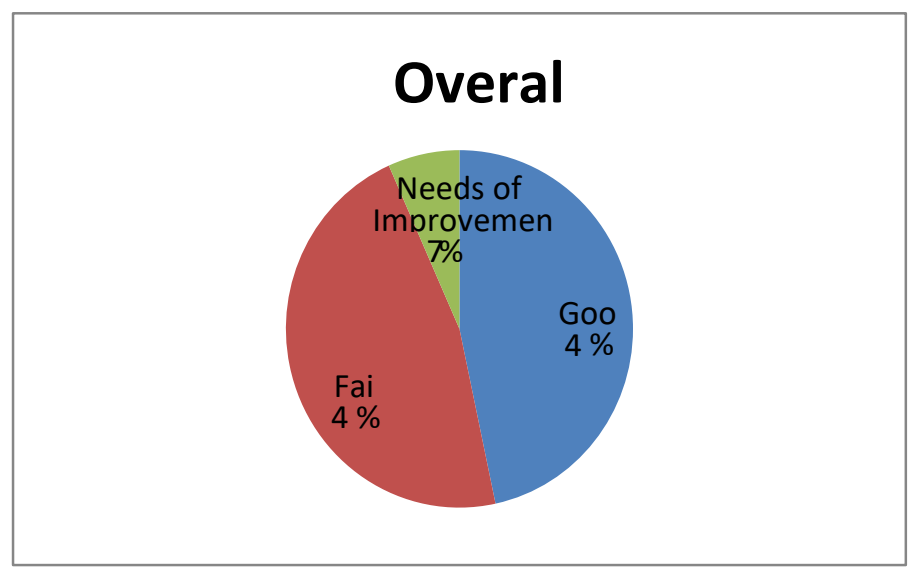

Figure 11 Overall analyses - pie chart

The overall section briefly describes the usefulness, accurateness, and how easy is the BSD application As the questionnaire, it was mostly fair as it ranked 47\%, a good $46 \%$ and needs of improvement 7\%, as showing in figure 10.

I really appreciated the comments and suggestions I've got out throughout the questionnaires, which gave me an opportunity to improve and satisfy the users. I went through all the comments, and maintained the suggest improvements, as well as it gave me a chance to fix the mistakes especially those audio ones.

\subsubsection{Operation and Maintenance:}

In the final phase, the system has been already completed, allocated and used. In this phase comments, suggestions and enquiries are given in order to gain benefits of the coming updates.

\section{Conclusion}

National slang is a valuable heritage that should always be preserved by any kind of means or methods. Online educations such as languages dictionaries have recently been used heavily due to the easiness and accessibility weather web based or mobile applications. Since technology becomes the main dominator in our lives thus we can utilize for those technological tools to preserve and protect our national slang and educate those who they been drafted away from their national slang.

\section{References}

[1].Kukulska-Hulme, A., \& Shield, L. (2008). An overview of mobile assisted language learning: From content delivery to supported collaboration and interaction. ReCALL, 20, 271-289.

[2].Vishal Chandr, Comparison between Various Software Development Methodologies, International Journal of Computer Applications (0975 - 8887), December 2015, Vol.131, No.9,

[3].Li Ma 1, Lei Gu2, Jin Wang3, “Research and Development of Mobile Application for Android platform”, International Journal of Multimedia and ubiquitous Engineering”, Nanjing ,2014, Vol.09, No. 4, pp187-198.

[4].[http://searchciomidmarket.techtarget.com/definition/HCI]

[5].Hochheiser,h., Lazar,J., HCI and Societal Issues: A Framework for Engagement, International Journal Of HumanComputer Interaction, 2007.

[6].[http://www.usask.ca/education/coursew ork/skaalid/theory/interface.htm]

[7].Carrie Williams, Research Methods, Journal of Business \& Economic Research , Grand Canyon University,

[8].March 2007, Volume 5, Number 3,

\section{Figure 1}

https://secweb.cs.odu.edu/ zeil/cs350/f14/Public/processModels/index.html 